\title{
Using SLIM-Based IMS-IMS Together with Cryogenic Infrared Spectroscopy for Glycan Analysis
}

Priyanka Bansal, Vasyl Yatsyna, Ali H. AbiKhodr, Stephan Warnke, Ahmed Ben Faleh, Natalia Yalovenko, Vicki H. Wysocki, and Thomas R. Rizzo*

Cite This: Anal. Chem. 2020, 92, 9079-9085

Read Online

ABSTRACT: The isomeric heterogeneity of glycans poses a great challenge for their analysis. While combining ion mobility spectrometry (IMS) with tandem mass spectrometry is a powerful means for identifying and characterizing glycans, it has difficulty distinguishing the subtlest differences between isomers. Cryogenic infrared spectroscopy provides an additional dimension for glycan identification that is extremely sensitive to their structure. Our approach to glycan analysis combines ultrahigh-resolution IMS-IMS using structures for lossless ion

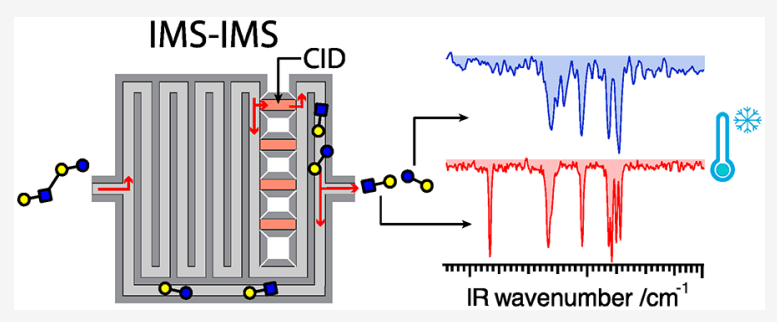
manipulation (SLIM) with cryogenic infrared spectroscopy. We present here the design of a SLIM board containing a series of on-board traps in which we perform collision-induced dissociation (CID) at pressures in the millibar range. We characterize the on-board CID process by comparing the fragments generated from a pentapeptide to those obtained on a commercial tandem mass spectrometer. We then apply our new technique to study the mobility and vibrational spectra of CID fragments from two human milk oligosaccharides. Comparison of both the fragment drift times and IR spectra with those of suitable reference compounds allows us to identify their specific isomeric form, including the anomericity of the glycosidic linkage, demonstrating the power of this tool for glycan analysis.

G lycans, or oligosaccharides, play major metabolic, physical, and structural roles in all biological systems. ${ }^{1}$ They mediate molecular interactions involved in immune response, inflammation, and cellular signaling, for example., ${ }^{2,3}$ Unfortunately, they possess inherent complexity that make them difficult to analyze. This complexity arises from the similarity of their isomeric monosaccharide building blocks as well as the variety of ways they can be attached. The monosaccharide units form a glycosidic linkage at a stereogenic carbon, leading to $\alpha$ and $\beta$ anomers. In addition, monosaccharides can form more than one glycosidic linkage, leading to regioisomers as well as the possibility of branched structures. Furthermore, the multiplicity of hydroxyl groups allows glycans to be functionalized at different positions, which leads to a distribution of positional isomers. Thus, to define completely the primary structure of a glycan, the monosaccharide composition, glycan sequence, anomericity of each glycosidic linkage, location of functionalization sites, and branching pattern must all be determined, and this requires extremely powerful tools.

Mass spectrometry (MS) is one of the most important methods for structural characterization of glycans due to its speed and sensitivity. ${ }^{4-6}$ While MS alone is unable to distinguish isomeric species, its combination with liquid chromatography $^{7,8}$ and enzyme degradation ${ }^{9}$ enables the resolution of many of these isomers. Moreover, tandem MS techniques $\left(\mathrm{MS}^{\mathrm{n}}\right)$ can provide detailed information including monosaccharide composition, connectivity, and branch- ing. ${ }^{10-15} \mathrm{~A}$ variety of dissociation techniques have been employed for tandem MS of glycans, including collision induced dissociation (CID), ${ }^{16,17}$ infrared multiphoton dissociation (IRMPD), ${ }^{18}$ and electron based methods. ${ }^{19-21}$ While CID is the most common method used in tandem MS, it induces fragmentation primarily at the glycosidic linkage in positively charged glycans. It has been largely assumed that cross-ring fragmentation is necessary to obtain information on the anomericity of the glycosidic bonds, and this has led to CID investigations of anionic glycans as well as the use of electron-based dissociation methods for cationic glycans. However, recent experiments from Compagnon and coworkers $^{22}$ as well as those by Pellegrinelli et al. ${ }^{23}$ have shown that the anomericity of glycosidic bonds seem to be retained in the $C_{n}$ fragments ${ }^{24}$ produced by CID, which renders this approach particularly powerful for glycan analysis.

Nevertheless, apart from relatively small oligosaccharides, $\mathrm{MS}^{\mathrm{n}}$ alone cannot distinguish among all isomeric forms. However, its limitations can be overcome by using MS in conjunction with additional orthogonal separation techniques.

Received: March 23, 2020

Accepted: May 27, 2020

Published: May 27, 2020 


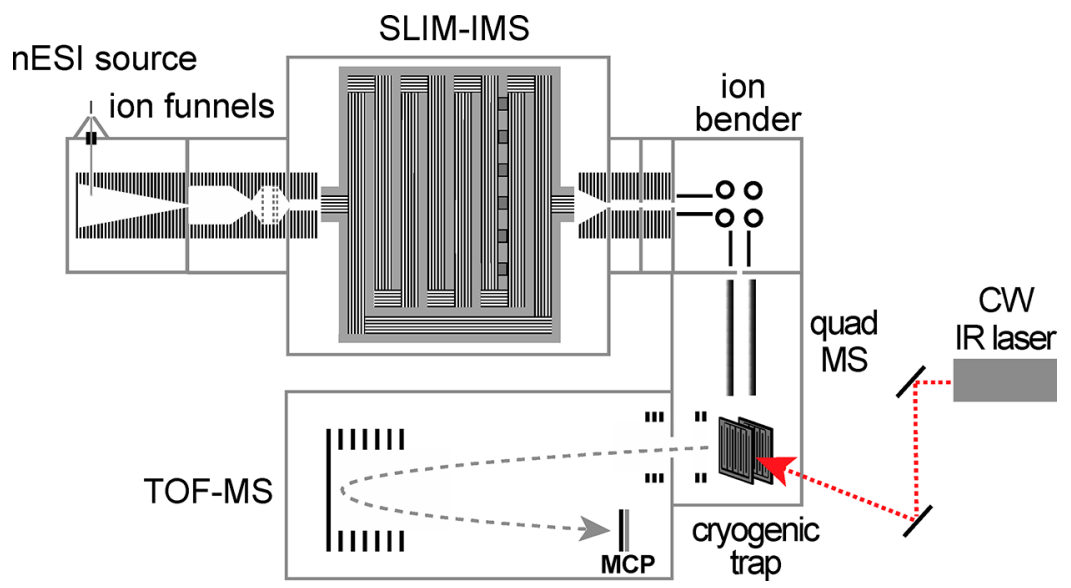

Figure 1. Schematic overview of the instrument.

Ion mobility spectrometry (IMS), which separates gas phase ions based on their average collision cross section (CCS), can be easily coupled to MS for glycan analysis. A particularly promising technique combines IMS with tandem MS, since the mobility of the fragments can be more informative than that of the intact parent ions. ${ }^{13,15,25-29}$ However, until recently, the available IMS techniques did not have sufficient resolving power for unambiguous identification of isomeric species. Using what they have termed structures for lossless ion manipulations (SLIM), Smith and co-workers have demonstrated ultrahigh-resolution ion mobility ${ }^{30-32}$ and applied it for glycan separation. ${ }^{33}$ In a similar approach, a cyclic IMS instrument has been recently developed to perform (IMS) ${ }^{\mathrm{n}}$ MS experiments and has been employed to study pentasaccharides. $^{34,35}$

For unambiguous identification of glycans, one can also add a spectroscopic dimension to IMS-MS. Vibrational spectroscopy in particular reveals important conformational and structural features, and many groups have combined it with MS for structural analysis of a variety of biomolecules, ${ }^{36,37}$ including glycans. ${ }^{38-41}$ While the use of infrared multiphoton dissociation (IRMPD) combined with MS has revealed anomer retention during CID of glycans, ${ }^{22}$ the limited spectral resolution at room temperature restricts its application to relatively small glycans and fragments. ${ }^{42}$ The spectral resolution can be improved by cryogenically cooling the ions, either in cold ion traps ${ }^{43-45}$ or in liquid helium droplets. ${ }^{46}$ Recently, ion mobility spectrometry has been combined with cryogenic infrared spectroscopy for identification and characterization of glycans, but the limited IMS resolution did not allow the separation of glycan isomers with subtle differences. ${ }^{38}$ A promising approach to overcome this limitation combines SLIM-based ultrahigh-resolution IMS with cryogenic infrared spectroscopy. ${ }^{43,44}$ Furthermore, isomeric glycans frequently produce isomeric fragments, and until now there has been no tool available to achieve isomer separation of the parent glycan prior to spectroscopic characterization of the fragments. We demonstrate here a new technique for the structural elucidation of glycans by combining ultrahighresolution, SLIM-based tandem IMS and cryogenic ion vibrational spectroscopy. This approach allows mobility separation of a parent glycan followed by CID, mobility separation of the fragments, and subsequent cryogenic IR spectroscopy of the fragments. This will enable the construction of a spectroscopic database for identifying fragments, which can then be used to help determine the parent glycan structure.

\section{EXPERIMENTAL METHODS}

SLIM-Based IMS Combined with Collision-Induced Dissociation. We perform experiments on a home-built instrument, shown schematically in Figure 1, which couples a SLIM-based traveling-wave ion mobility device with a cryogenic ion trap for spectroscopy and a time-of-flight (TOF) mass spectrometer. ${ }^{43}$ Briefly, the ions produced by a nanoelectrospray source are transferred into the instrument through a stainless-steel capillary heated to $100-150{ }^{\circ} \mathrm{C}$. A dual-stage ion funnel assembly (MassTech) accumulates the ions before releasing them in short packets $(\sim 150 \mu \mathrm{s})$ into a SLIM-based ion-mobility region, where they are separated by their shape (i.e., their rotationally averaged collisional cross section). The arrival-time distributions (ATDs) of the ions can be acquired on a channeltron detector either after $\mathrm{m} / z$ selection by a quadrupole mass filter placed after the IMS region, or without $m / z$ selection when the arrival times of CID fragments are of interest. The SLIM device, described in detail below, includes a section explicitly designed for collisioninduced dissociation. After IMS ${ }^{\mathrm{n}}$ analysis, the ions of interest are directed through the differential pumping stages of the instrument using ring-electrode and hexapole guides before entering the cryogenic ion trap, maintained at $40 \mathrm{~K}$, where they are confined and cooled by collisions with a cold buffer gas composed of $\mathrm{He} / \mathrm{N}_{2}$ in a 90:10 mixture. During the cooling process the ions are tagged with weakly bound $\mathrm{N}_{2}$ molecules that serve as a messenger to detect IR absorption. The tagged ions are irradiated for $50 \mathrm{~ms}$ with a continuous-wave mid-IR laser (IPG Photonics) operated with $0.2 \mathrm{~W}$ output power. Absorption of an infrared photon leads to the redistribution of vibrational energy, causing the $\mathrm{N}_{2}$ tag to boil off. We then extract the ions into a TOF-MS and obtain an IR spectrum of the species by monitoring the number of tagged ions as a function of the laser wavenumber.

Ion mobility separation of ions is achieved using a SLIM device that we constructed following the work of Smith and coworkers. ${ }^{30,47}$ Here, traveling-wave (TW) potentials for IM separation are created between a "sandwich" of two mirrored printed circuit boards (PCBs), where RF and DC fields are used for ion confinement, and traveling square-well potentials propel the ions through the $\mathrm{N}_{2}$ drift gas at a pressure of 1.5 mbar (see Figure S1). We recently implemented this technique 

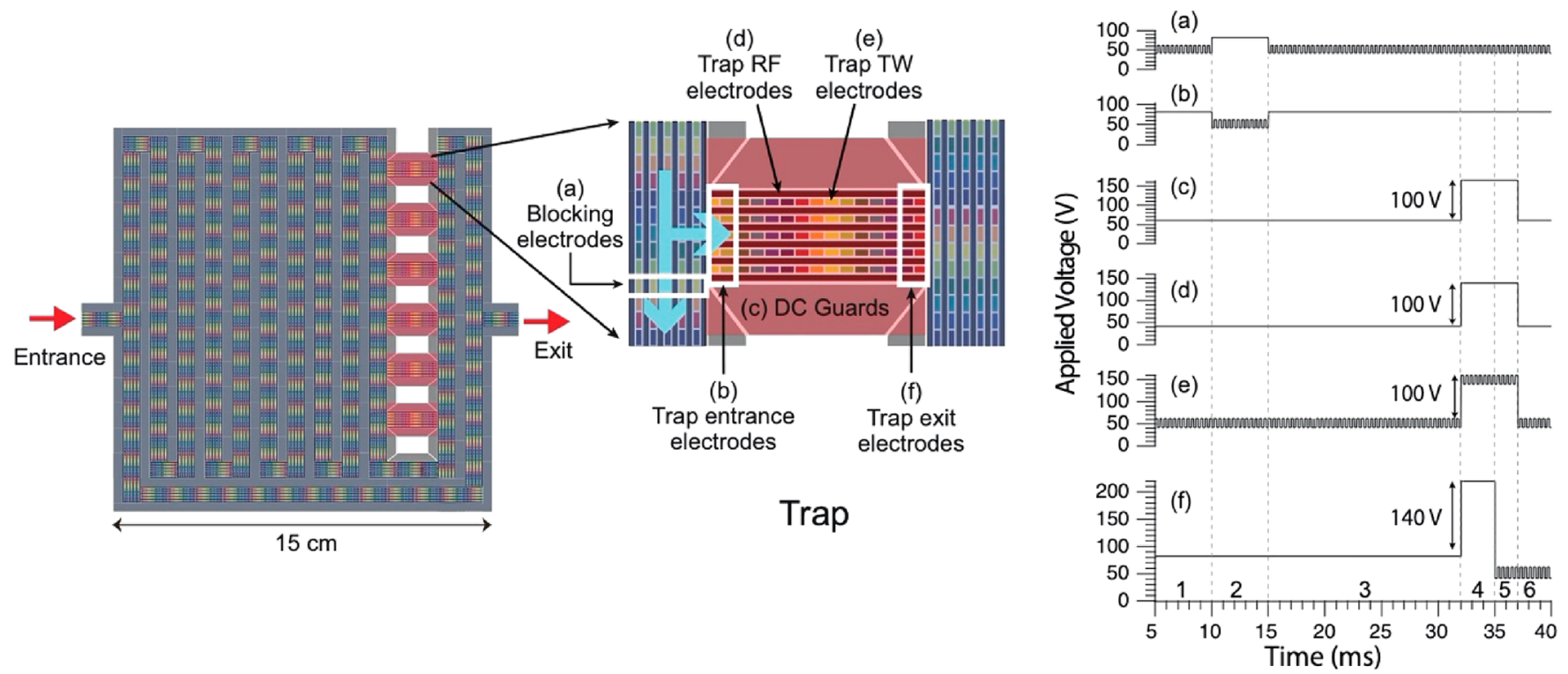

Figure 2. Layout of SLIM-IMS-CID device. Left: overview of SLIM board. Center: details of on-board traps used for CID. Right: potentials used for isolating, trapping, and performing CID in the trap section of the SLIM board. Periods for mobility separation (1), trap filling (2), ion trapping (3), bias increase (4), ion release and fragmentation (5), and further separation or exiting the board (6) are indicated. A more detailed view of the SLIM electrodes is given in Figure S1.

using a $15 \mathrm{~cm} \times 15 \mathrm{~cm}$ device with a single-cycle path length of $1.8 \mathrm{~m} .{ }^{43}$ Longer path lengths can be achieved by allowing the ions to traverse multiple cycles between the SLIM boards. ${ }^{31,43}$

In the present work, we modified the design of our previously published SLIM-IMS device ${ }^{43}$ to incorporate a section in which we perform on-board CID, shown in Figure 2. In addition to the $1.5 \mathrm{~m}$ serpentine track for ion separation, our new device has a series of on-board traps for selection, storage, and fragmentation of mobility-separated ions, ${ }^{48}$ one of which is depicted in the inset of Figure 2. The procedure by which the ions are fragmented can be explained with the help of the timing diagram displayed in the right-hand panel of Figure 2, which shows the voltages applied to various electrodes. During period 1, precursor ions are separated on the serpentine track by a traveling wave of $20 \mathrm{~V}$ amplitude. When a packet of precursor ions of a certain mobility approaches the trap entrance, we steer them into the trap during period 2 by applying a $40 \mathrm{~V} \mathrm{DC}$ bias to the blocking electrodes (trace a) while simultaneously lowering the bias on the trap entrance electrodes (trace b) and applying a traveling wave to them. After the parent ions of interest enter the trap, the entrance electrodes are blocked with a $40 \mathrm{~V}$ bias. The trap exit electrodes are maintained at $40 \mathrm{~V}$ bias to store the ions until fragmentation conditions are applied. During period 3 in which precursor ions are stored in the trap, a TW continually pushes the ions toward the exit electrodes.

In order to perform fragmentation, $3 \mathrm{~ms}$ before ions are released, the bias potential of all electrodes in the trap (i.e., DC guard, RF, and TW electrodes) are raised during period 4 by $100 \mathrm{~V}$ (traces $\mathrm{c}-\mathrm{e}$, respectively), while the exit electrode bias is raised by $140 \mathrm{~V}$. In order to release and accelerate the stored ions, the TW wave is applied to the exit electrodes simultaneously with lowering their bias potential by $180 \mathrm{~V}$ (trace $\mathrm{f}$ and period 5). The potential difference of $100 \mathrm{~V}$ between the trap and the normal serpentine track accelerates ions, which are energized by collisions with the drift gas and fragmented as they exit the trap. The fragments created in this way follow the TW toward the exit of the SLIM device and are either recycled for additional mobility separation (period 6) or diverted to the cryogenic trap, where we record a vibrational spectrum. Further details of the SLIM device and its operating parameters are given in the Supporting Information.

Materials. Oligosaccharides $N$-acetyl-D-lactosamine (Gal $\beta 1-4 \mathrm{GlcNAc})$, lacto- $N$-neotetraose (LNnT), and lacto$N$-tetraose (LNT) were purchased from Dextra (U.K.), while lacto- $N$-hexaose (LNH) was purchased from Carbosynth (U.K.). All were used without further purification. For nanoelectrospray, the pentapeptide AAPPA, Gal $\beta 1-4 \mathrm{GlcNAc}$, LNnT and LNT samples were prepared in a 50:50 solution of water/methanol, and $\mathrm{LNH}$ was prepared in a 30:70 solution of water/acetonitrile to yield a concentration of $10-30 \mu \mathrm{M}$. All solvents were HPLC grade. The experiments were performed using nitrogen $\left(\mathrm{N}_{2}\right)$ as both a drift gas for ion mobility and a collision gas for CID.

\section{RESULTS AND DISCUSSION}

Characterization of On-Board CID. The first aim of this study was to characterize on-board CID, which we evaluated using the pentapeptide AAPPA. The singly charged, protonated peptide ions $[\mathrm{AAPPA}+\mathrm{H}]^{+}(\mathrm{m} / z=426.4 \mathrm{Da})$ were separated from unwanted ions by ion mobility and loaded into the on-board trap. The fragmentation pattern generated upon raising the trap bias and ejecting parent ions is compared to that obtained on a commercial Q-TOF (Waters Premier) in Figure 3. In both cases, the major fragments observed upon CID are the $b_{3}, b_{4}$, and $y_{4}$ ions as well as the internal fragments AP, PP and APP. As shown in the figure, the fragmentation pattern for AAPPA in the SLIM trap is similar to that obtained on the Q-TOF with ions accelerated into the collision region with $20-25 \mathrm{eV}$.

IMS-IMS Combined with Cryogenic Infrared Spectroscopy. Having gained the ability to fragment ions directly on the SLIM board, we then applied this technique to characterize glycan fragmentation, starting with the human milk oligosaccharide LNnT. Singly charged, sodiated LNnT ions $(m / z=730.2 \mathrm{Da})$ were loaded into the trap and 


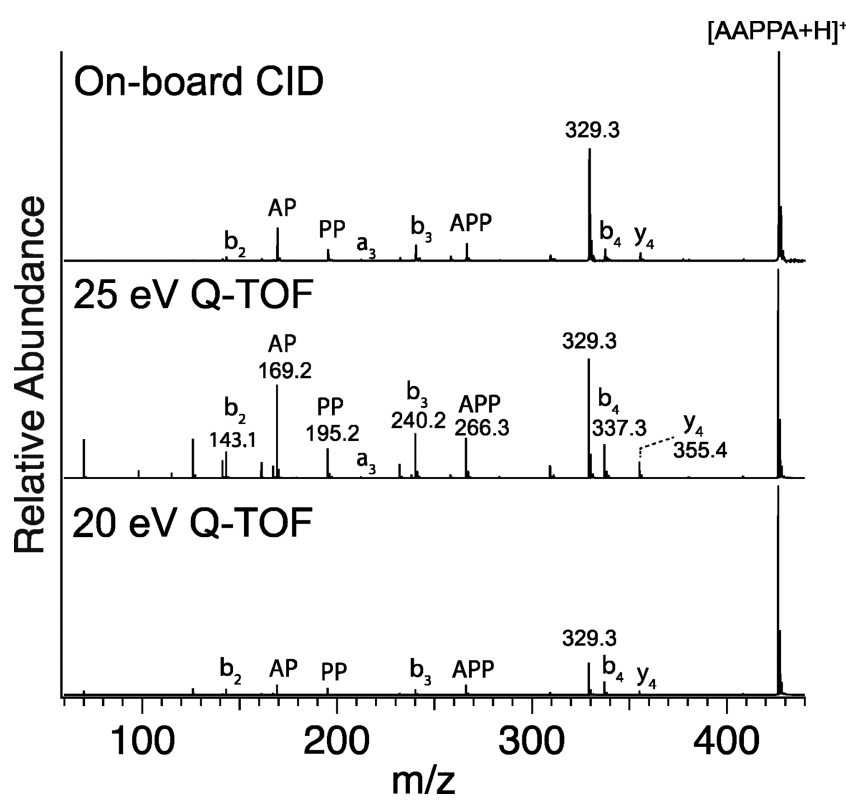

Figure 3. Comparison of CID MS-MS spectra of singly charged, protonated peptide AAPPA obtained by on-board CID and Q-TOF Premier, both of which use $\mathrm{N}_{2}$ as a collision gas.

fragmented as described above. The bottom trace of Figure 4a shows the ATD of parent ions that are trapped for $55 \mathrm{~ms}$ and then sent through an additional cycle on the SLIM board before exiting and being detected. The upper trace shows the

(a)

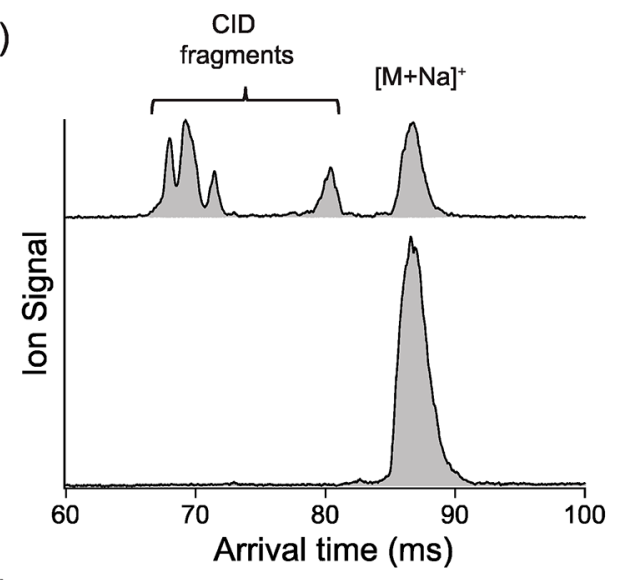

(b)

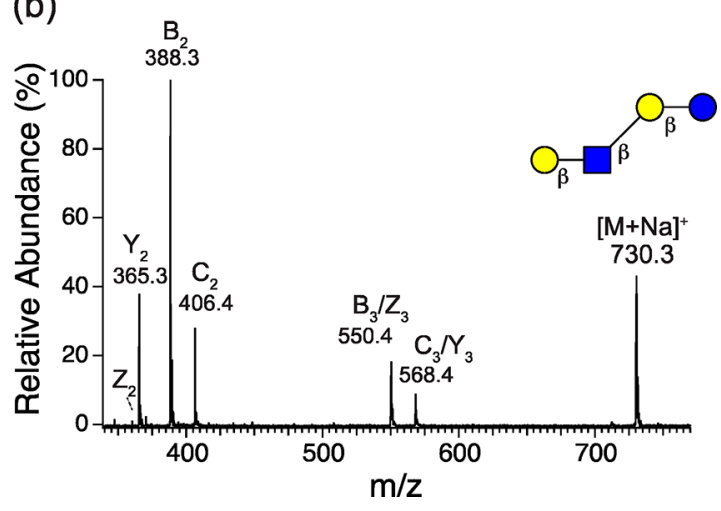

Figure 4. (a) ATD of the $[\mathrm{LNnT}+\mathrm{Na}]^{+}$with and without fragmentation, on the top and bottom, respectively, after the ions exit the trap and undergo one additional SLIM cycle. (b) Mass spectrum of $[\mathrm{LNnT}+\mathrm{Na}]^{+}$and its CID fragments. result using the same timing, but this time raising the bias potential of the trap before ejecting ions back onto the separation track. Fragmentation of the parent glycan ions as they exit the trap results in fragments that are separated from the parent during the additional SLIM cycle after CID.

In this case, four well-resolved fragment peaks arrive earlier than the parent ions. The mass spectrum (Figure $4 \mathrm{~b}$ ) was recorded by sending all of the ions in the arrival-time distribution to the TOF mass spectrometer. Fragmentation occurs mainly at the glycosidic linkages, and the major fragment produced is the $\mathrm{B}_{2}$ fragment at $\mathrm{m} / z$ 388.3 $\mathrm{Da}$ along with other $\mathrm{B} / \mathrm{Y}$ fragments $\left(\mathrm{B}_{3}, \mathrm{Y}_{3}, \mathrm{Y}_{4}\right){ }^{24}$ The $\mathrm{C} / \mathrm{Z}\left(\mathrm{C}_{2}, \mathrm{Z}_{2}\right)$ fragments appear but are much less abundant. The $C$ fragments correspond to an intact glycan and may contain information about the anomericity of glycosidic linkages, and for this reason we focused on the $\mathrm{C}_{2}$ fragment.

Anomeric Memory during Breakage of the Glycosidic Linkage. To address the question of whether anomericity is retained upon fragmentation, we used the intact disaccharide (Gal $\beta 1-4 \mathrm{GlcNAc})$, which corresponds to the $\mathrm{C}_{2}$ fragment from $\mathrm{LNnT}$, as a reference. To be able to compare the ATD of the reference compound with that of the $\mathrm{C}_{2}$ fragment, it is important that they follow exactly the same path on the SLIM device. For this reason, the disaccharide reference ions are first isolated and loaded into the trap, where they remain for few milliseconds, and then subjected to a few additional separation cycles after exiting the trap, which is precisely what was done for the $\mathrm{C}_{2}$ fragments. As shown in Figure 5a (top), the ATD of (a)

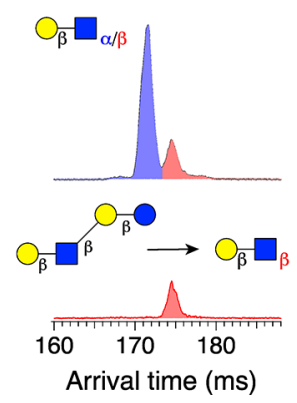

(b)

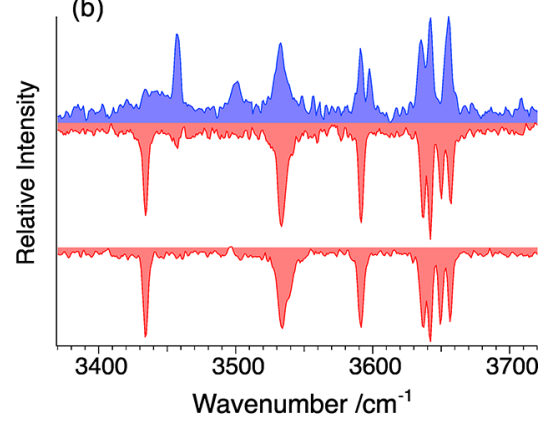

Figure 5. (a) ATD of reference sugar Gal $\beta 1-4 \mathrm{GlcNAc}$ (top) and the $\mathrm{C}_{2}$ fragment (bottom) generated upon $\mathrm{CID}$ of $[\mathrm{LNnT}+\mathrm{Na}]^{+}$, both obtained after 9 SLIM cycles. (b) The two upper traces represent the cryogenic IR spectrum of the mobility-separated anomers of Gal $\beta 1-$ 4GlcNAc, and the bottom trace represents the IR spectrum of the $\mathrm{C}_{2}$ fragment.

the reference compound exhibits two peaks after nine cycles on the SLIM board. The observation of two peaks in the ATD seems to be characteristic of reducing sugars. ${ }^{33,44}$ These peaks have been attributed to the $\alpha$ and $\beta$ anomers of the monosaccharide at the reducing end, and their intensities reflect the $\alpha / \beta$ equilibrium ratio in solution. ${ }^{49}$ The observation of only a single mobility peak in the $\mathrm{C}_{2}$ fragments from LNnT (Figure 5a (bottom)), suggests that only one of the two anomers is present after fragmentation. To confirm this, the spectroscopic fingerprint of both mobility peaks of the $\mathrm{Gal} \beta 1-$ 4GlcNAc reference were recorded and compared to that of the $\mathrm{C}_{2}$ fragment, as shown in Figure $5 \mathrm{~b}$. It is clear that the IR spectrum of the $\mathrm{C}_{2}$ fragment corresponds to that of the second drift peak. ${ }^{23}$ These results clearly confirm the retention of anomericity under the CID conditions used in this work. In 


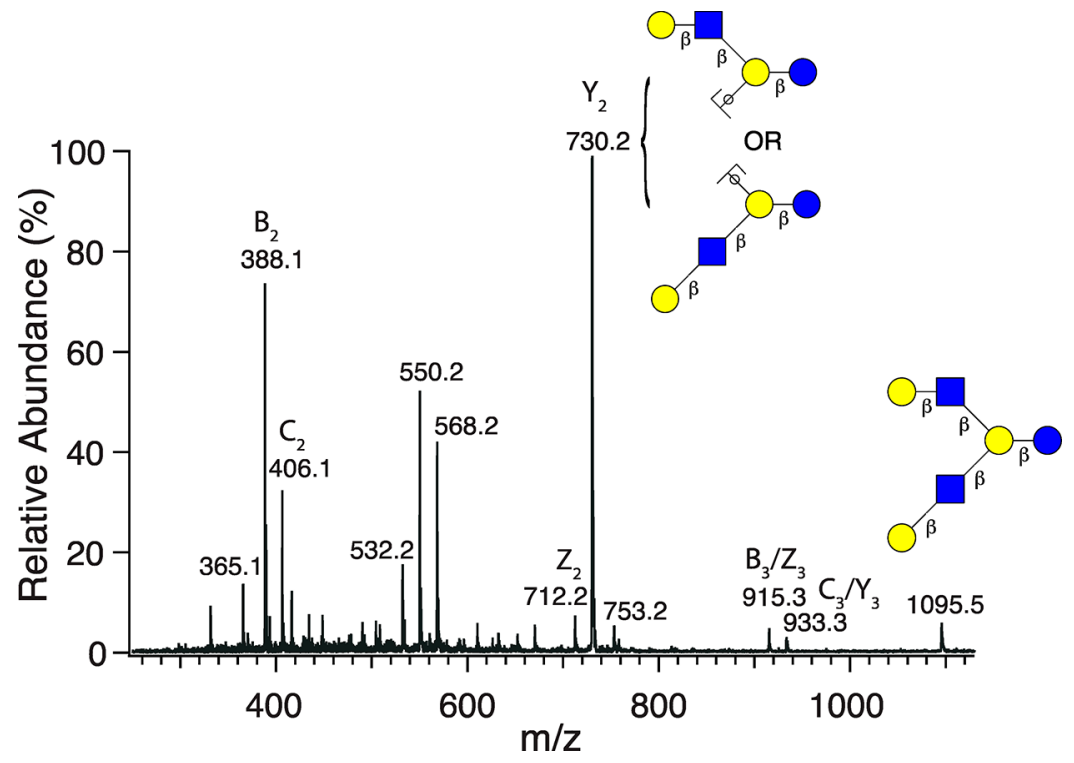

Figure 6. CID mass spectrum of $[\mathrm{LNH}+\mathrm{Na}]^{+}$obtained by on-board CID.

previous CID experiments on $\mathrm{LNnT}$ performed at low pressure and without mobility analysis, ${ }^{23}$ we demonstrated on the basis of its vibrational spectrum alone that the $\mathrm{C}_{2}$ fragment corresponds to only one of the two species that we observe upon high-resolution mobility separation of the $\mathrm{Gal} \beta 1-4 \mathrm{GlcNAc}$ reference compound. In this work where we perform CID at millibar pressures, we not only obtain the same spectrum as Pellegrinelli et al.; ${ }^{23}$ we also measure the mobility of the fragment and see that it matches to only one of the two mobility-separated peaks of the reference compound.

In the case of the disaccharide GalNac $\alpha 1-3 \mathrm{Gal}$, we previously assigned the absolute configuration of the anomeric $\mathrm{OH}$ using anomerically pure, methylated reference compounds together with oxygen-18 subtitution. ${ }^{44}$ While we have not done this in the case of $\mathrm{Gal} \beta 1-4 \mathrm{GlcNAc}$, the fact that we break a $\beta$ glycosidic linkage and observe only a single anomer rather than a mixture strongly suggests that the slower mobility peak in Figure 5a corresponds to the $\beta$ anomer and the faster one to the $\alpha$ anomer. It seems unlikely that the configuration about the $\mathrm{C} 1$ carbon of $\mathrm{Gal} \beta 1-4 \mathrm{GlcNAc}$ would invert with $100 \%$ yield from $\beta$ to $\alpha$ upon CID, although technically, we cannot rule it out without using anomerically pure reference compounds.

Isomeric Fragment Identification. Oligosaccharide sequencing is challenging not only because of the presence of various isomeric forms of the parent molecule, but also because the fragments can themselves be isomeric.

Figure 6 shows the mass spectrum of the hexasaccharide LNH (singly sodiated, $m / z=1095.2 \mathrm{Da}$ ) upon CID on our SLIM board. The $\mathrm{Y}_{2}$ fragment, which consists of an intact glycan of $m / z 730.2 \mathrm{Da}$, can arise from two different fragmentation paths. The LNH precursor can either lose the top branch, yielding the tetrasaccharide $\mathrm{Gal} \beta 1-3 \mathrm{GlcNac} \beta 1-$ $3 \mathrm{Gal} \beta 1-4 \mathrm{Glc}$, which is called lacto-N-tetraose (LNT), or the bottom branch yielding $\mathrm{Gal} \beta 1-4 \mathrm{GlcNac} \beta 1-6 \mathrm{Gal} \beta 1-4 \mathrm{Glc}$, which is not commercially available as a standard. Both fragments have the same composition, corresponding to $\mathrm{m} / \mathrm{z}$ $730.2 \mathrm{Da}$ (in the sodiated form) and only differ in the configuration of two glycosidic bonds. To be able to use tandem mass spectrometry to reconstruct the parent glycan structure in such cases, we need to be able to distinguish these types of isomeric fragments. To do so, we allowed these fragment ions to undergo additional SLIM cycles after they exit from the trap. The single peak at $\mathrm{m} / z$ of $730.2 \mathrm{Da}$ separates into three different peaks in the ATD, shown on the bottom left of Figure 7 . These peaks could correspond to the different

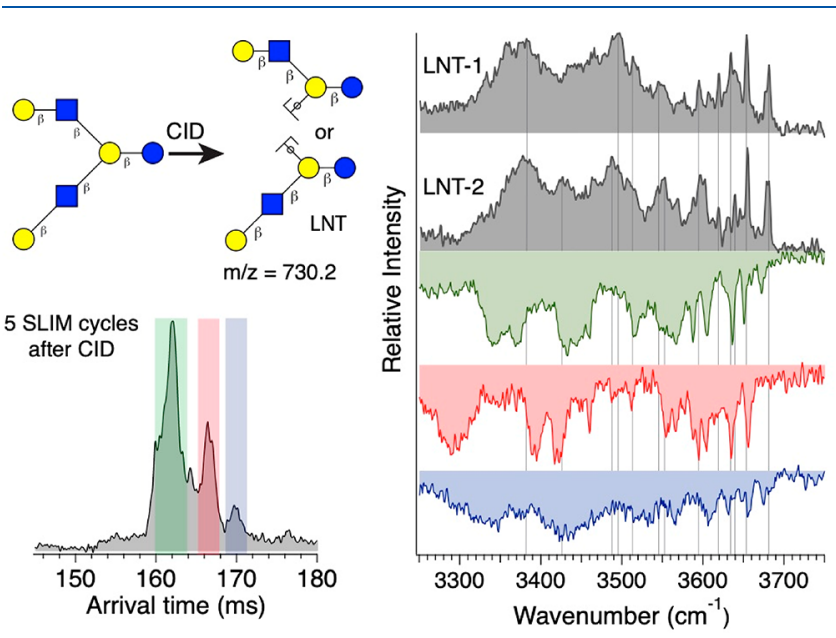

Figure 7. Arrival time distribution of the $\mathrm{Y}_{2}$ fragment of $\mathrm{m} / \mathrm{z} 730.2$ formed upon fragmentation of LNH. The cryogenic IR spectrum of mobility-separated drift peaks is shown below (green, pink, and blue trace for first, second, and third peak respectively). The gray traces show the IR spectra of the LNT reference for comparison.

isomeric fragments or to conformers of a single fragment. To investigate this, we recorded the cryogenic IR spectrum of each of the mobility separated peaks at $m / z 730.2 \mathrm{Da}$ and compared it to those of tetrasaccharide LNT, which would be the fragment produced from CID of LNH if it would lose the upper branch. The ATD of LNT has two peaks (Figure S2), and we measure the spectrum of each of them separately. One can clearly see that none of the measured spectra corresponds to that of either isomer of LNT, which suggests that the $Y_{2}$ fragment results from loss of the lower branch. Indeed, Pfenninger et al. reported a similar result for CID of LNH in negative ion mode. ${ }^{50}$ While we have excluded the possibility 
that $\mathrm{Y}_{2}$ corresponds to LNT, further experiments are needed to characterize the nature of the species that give rise to the observed ATD features and spectra. Nevertheless, the data of Figure 7 demonstrates the ability of our new technique to distinguish between isomeric fragments, making it a powerful tool for glycan sequencing.

\section{CONCLUSION}

We have demonstrated a new approach for glycan identification by combining IMS-IMS with cryogenic IR spectroscopy. The first results illustrate the ability of this tool to address fundamental questions regarding the fragmentation mechanism of the glycosidic linkage. In the case of the tetrasaccharide LnNT, we found that the anomeric configuration of the glycosidic bond is preserved upon generation of the $\mathrm{C}_{2}$ fragment; we could make this assignment on the basis of either the fragment ATD or its cryogenic vibrational spectrum. We also used this new tool to study a larger glycan, $\mathrm{LNH}$, where there is a possibility to generate isomeric fragments. Ultrahigh-resolution IMS combined with cryogenic vibrational spectroscopy shows that at least three distinct isomers of the $\mathrm{Y}_{2}$ fragment of $\mathrm{LNH}$ exist, and that none of them result from the loss of the upper branch.

More generally, having the ability to separate isomeric fragments based upon high-resolution ion mobility allows us to record their spectroscopic fingerprint, and this is particularly important for those for which standards are not available commercially. Our ultimate goal is to develop an $(\mathrm{IMS})^{\mathrm{n}}$ technique combined with cryogenic infrared spectroscopy, where a parent glycan of unknown structure is first mobility selected and fragmented. The fragments then can be retrapped, fragmented further, and mobility separated such that an IR spectrum could be obtained on the next generation of fragments. This approach would help us to create a complete database of all possible fragments, which in the case of $C_{n}$ fragments, provides information about the stereochemistry of glycosidic bonds. Such a database should facilitate reconstruction of the parent glycan structure. Once this is determined, the vibrational spectrum of the parent glycan can then be put in a database and used for identification.

\section{ASSOCIATED CONTENT}

\section{SI Supporting Information}

The Supporting Information is available free of charge at https://pubs.acs.org/doi/10.1021/acs.analchem.0c01265.

Operating parameters for the SLIM-based ion mobility separation device in which collision induced dissociation is carried out and arrival-time distribution (ATD) of the LNT reference compound (PDF)

\section{AUTHOR INFORMATION}

\section{Corresponding Author}

Thomas R. Rizzo - Laboratoire de Chimie Physique Moleculaire, Ecole Polytechnique Féderale de Lausanne, CH1015 Lausanne, Switzerland; 이이.org/0000-0003-2796905X; Email: thomas.rizzo@epfl.ch

\section{Authors}

Priyanka Bansal - Laboratoire de Chimie Physique Moleculaire, Ecole Polytechnique Féderale de Lausanne, CH-1015 Lausanne, Switzerland
Vasyl Yatsyna - Laboratoire de Chimie Physique Moleculaire, Ecole Polytechnique Féderale de Lausanne, CH-1015 Lausanne, Switzerland; Department of Physics, University of Gothenburg, 41296 Gotheburg, Sweden

Ali H. AbiKhodr - Laboratoire de Chimie Physique Moleculaire, Ecole Polytechnique Féderale de Lausanne, $\mathrm{CH}$ 1015 Lausanne, Switzerland

Stephan Warnke - Laboratoire de Chimie Physique Moleculaire, Ecole Polytechnique Féderale de Lausanne, $\mathrm{CH}$ 1015 Lausanne, Switzerland

Ahmed Ben Faleh - Laboratoire de Chimie Physique Moleculaire, Ecole Polytechnique Féderale de Lausanne, $\mathrm{CH}$ 1015 Lausanne, Switzerland

Natalia Yalovenko - Laboratoire de Chimie Physique Moleculaire, Ecole Polytechnique Féderale de Lausanne, $\mathrm{CH}$ 1015 Lausanne, Switzerland

Vicki H. Wysocki - Department of Chemistry and Biochemistry, The Ohio State University, Columbus, Ohio 43210, United States; orcid.org/0000-0003-0495-2538

Complete contact information is available at:

https://pubs.acs.org/10.1021/acs.analchem.0c01265

\section{Notes}

The authors declare no competing financial interest.

\section{ACKNOWLEDGMENTS}

The authors thank the Swiss National Science Foundation through Grant 200020 184838, the European Research Council (Grant 788697-GLYCANAL), the Swedish Research Council (international postdoc Grant 2019-00512), and the EPFL for the financial support of this work. We also thank Erin Panczyk of OSU for synthesis of the AAPPA and NIH R01 GM113658 to V.H.W. Finally, we thank Gabe Nagy of PNNL for helpful discussions regarding CID on SLIM.

\section{REFERENCES}

(1) Varki, A. Glycobiology 2017, 27 (1), 3-49.

(2) Cummings, R. D.; Pierce, J. M. Chem. Biol. 2014, 21 (1), 1-15.

(3) Dwek, R. A. Chem. Rev. 1996, 96 (2), 683-720.

(4) An, H. J.; Lebrilla, C. B. Mass Spectrom. Rev. 2011, 30 (4), 560578.

(5) Mechref, Y.; Novotny, M. V.; Krishnan, C. Anal. Chem. 2003, 75

(18), 4895-4903.

(6) Wuhrer, M. Glycoconjugate J. 2013, 30 (1), 11-22.

(7) Liu, S.; Gao, W.; Wang, Y.; He, Z.; Feng, X.; Liu, B.-F.; Liu, X. PLoS One 2017, 12 (1), e0170013.

(8) Shah, B.; Jiang, X. G.; Chen, L.; Zhang, Z. J. Am. Soc. Mass Spectrom. 2014, 25 (6), 999-1011.

(9) Morelle, W.; Michalski, J.-C. Nat. Protoc. 2007, 2 (7), 15851602.

(10) Harvey, D. J.; Abrahams, J. L. Rapid Commun. Mass Spectrom. 2016, 30 (5), 627-634.

(11) Harvey, D. J.; Jaeken, J.; Butler, M.; Armitage, A. J.; Rudd, P. M.; Dwek, R. A. J. Mass Spectrom. 2010, 45 (5), 528-535.

(12) Harvey, D. J.; Rudd, P. M. Int. J. Mass Spectrom. 2011, 305 (23), $120-130$

(13) Harvey, D. J.; Seabright, G. E.; Vasiljevic, S.; Crispin, M.; Struwe, W. B. J. Am. Soc. Mass Spectrom. 2018, 29 (5), 972-988.

(14) Harvey, D. J.; Struwe, W. B. J. Am. Soc. Mass Spectrom. 2018, 29 (6), 1179-1193.

(15) Hofmann, J.; Hahm, H. S.; Seeberger, P. H.; Pagel, K. Nature 2015, 526 (7572), 241-244.

(16) Lemoine, J.; Fournet, B.; Despeyroux, D.; Jennings, K. R.; Rosenberg, R.; Hoffmann, E. J. Am. Soc. Mass Spectrom. 1993, 4 (3), 197-203. 
(17) Reinhold, V. N.; Reinhold, B. B.; Costello, C. E. Anal. Chem. 1995, 67 (11), 1772-1784.

(18) Lancaster, K. S.; An, H. J.; Li, B.; Lebrilla, C. B. Anal. Chem. 2006, 78 (14), 4990-4997.

(19) Han, L.; Costello, C. E. J. Am. Soc. Mass Spectrom. 2011, 22 (6), 997-1013.

(20) Wolff, J. J.; Chi, L.; Linhardt, R. J.; Amster, I. J. Anal. Chem. 2007, 79 (5), 2015-2022.

(21) Zhao, C.; Xie, B.; Chan, S.-Y.; Costello, C. E.; O’Connor, P. B. J. Am. Soc. Mass Spectrom. 2008, 19 (1), 138-150.

(22) Schindler, B.; Barnes, L.; Renois, G.; Gray, C.; Chambert, S.; Fort, S.; Flitsch, S.; Loison, C.; Allouche, A.-R.; Compagnon, I. Nat. Commun. 2017, 8 (1), 1-7.

(23) Pellegrinelli, R. P.; Yue, L.; Carrascosa, E.; Warnke, S.; Ben Faleh, A.; Rizzo, T. R. J. Am. Chem. Soc. 2020, 142, 5948.

(24) Domon, B.; Costello, C. E. Glycoconjugate J. 1988, 5 (4), 397409.

(25) Merenbloom, S. I.; Koeniger, S. L.; Valentine, S. J.; Plasencia, M. D.; Clemmer, D. E. Anal. Chem. 2006, 78 (8), 2802-2809.

(26) Harvey, D. J.; Scarff, C. A.; Edgeworth, M.; Crispin, M.; Scanlan, C. N.; Sobott, F.; Allman, S.; Baruah, K.; Pritchard, L.; Scrivens, J. H. Electrophoresis 2013, 34 (16), 2368-2378.

(27) Harvey, D. J.; Watanabe, Y.; Allen, J. D.; Rudd, P.; Pagel, K.; Crispin, M.; Struwe, W. B. J. Am. Soc. Mass Spectrom. 2018, 29 (6), $1250-1261$.

(28) Hinneburg, H.; Hofmann, J.; Struwe, W. B.; Thader, A.; Altmann, F.; Varón Silva, D.; Seeberger, P. H.; Pagel, K.; Kolarich, D. Chem. Commun. 2016, 52 (23), 4381-4384.

(29) Pu, Y.; Ridgeway, M. E.; Glaskin, R. S.; Park, M. A.; Costello, C. E.; Lin, C. Anal. Chem. 2016, 88 (7), 3440-3443.

(30) Deng, L.; Ibrahim, Y. M.; Hamid, A. M.; Garimella, S. V. B.; Webb, I. K.; Zheng, X.; Prost, S. A.; Sandoval, J. A.; Norheim, R. V.; Anderson, G. A.; Tolmachev, A. V.; Baker, E. S.; Smith, R. D. Anal. Chem. 2016, 88 (18), 8957-8964.

(31) Deng, L. L.; Webb, I. K.; Garimella, S. V. B.; Hamid, A. M.; Zheng, X. Y.; Norheim, R. V.; Prost, S. A.; Anderson, G. A.; Sandoval, J. A.; Baker, E. S.; Ibrahim, Y. M.; Smith, R. D. Anal. Chem. 2017, 89 (8), 4628-4634.

(32) Ibrahim, Y. M.; Hamid, A. M.; Deng, L.; Garimella, S. V. B.; Webb, I. K.; Baker, E. S.; Smith, R. D. Analyst 2017, 142 (7), 10101021.

(33) Nagy, G.; Attah, I. K.; Garimella, S. V. B.; Tang, K.; Ibrahim, Y. M.; Baker, E. S.; Smith, R. D. Chem. Commun. (Cambridge, U. K.) 2018, 54 (83), 11701-11704.

(34) Giles, K.; Ujma, J.; Wildgoose, J.; Pringle, S.; Richardson, K.; Langridge, D.; Green, M. Anal. Chem. 2019, 91 (13), 8564-8573.

(35) Ujma, J.; Ropartz, D.; Giles, K.; Richardson, K.; Langridge, D.; Wildgoose, J.; Green, M.; Pringle, S. J. Am. Soc. Mass Spectrom. 2019, 30 (6), 1028-1037.

(36) Rijs, A. M.; Oomens, J. Gas-Phase IR Spectroscopy and Structure of Biological Molecules. Springer International Publishing: Berlin, 2015.

(37) Rizzo, T.; Boyarkin, O. Cryogenic Methods for the Spectroscopy of Large, Biomolecular Ions. In Gas-Phase IR Spectroscopy and Structure of Biological Molecules; Rijs, A. M., Oomens, J., Eds.; Springer International Publishing: Berlin, 2015; Vol. 364, pp 43-97.

(38) Masellis, C.; Khanal, N.; Kamrath, M. Z.; Clemmer, D. E.; Rizzo, T. R. J. Am. Soc. Mass Spectrom. 2017, 28 (10), 2217-2222.

(39) Polfer, N. C.; Valle, J. J.; Moore, D. T.; Oomens, J.; Eyler, J. R.; Bendiak, B. Anal. Chem. 2006, 78 (3), 670-679.

(40) Depraz Depland, A.; Renois-Predelus, G.; Schindler, B.; Compagnon, I. Int. J. Mass Spectrom. 2018, 434, 65-69.

(41) Rabus, J. M.; Simmons, D. R.; Maitre, P.; Bythell, B. J. Phys. Chem. Chem. Phys. 2018, 20 (44), 27897-27909.

(42) Oomens, J.; Sartakov, B. G.; Meijer, G.; von Helden, G. Int. J. Mass Spectrom. 2006, 254 (1-2), 1-19.

(43) Ben Faleh, A.; Warnke, S.; Rizzo, T. R. Anal. Chem. 2019, 91 (7), 4876-4882.

(44) Warnke, S.; Ben Faleh, A.; Scutelnic, V.; Rizzo, T. R. J. Am. Soc. Mass Spectrom. 2019, 30 (11), 2204-2211.
(45) Warnke, S.; Ben Faleh, A.; Pellegrinelli, R. P.; Yalovenko, N.; Rizzo, T. R. Faraday Discuss. 2019, 217 (0), 114-125.

(46) Mucha, E.; González Flórez, A. I.; Marianski, M.; Thomas, D. A.; Hoffmann, W.; Struwe, W. B.; Hahm, H. S.; Gewinner, S.; Schöllkopf, W.; Seeberger, P. H.; von Helden, G.; Pagel, K. Angew. Chem., Int. Ed. 2017, 56 (37), 11248-11251.

(47) Hamid, A. M.; Garimella, S. V. B.; Ibrahim, Y. M.; Deng, L.; Zheng, X.; Webb, I. K.; Anderson, G. A.; Prost, S. A.; Norheim, R. V.; Tolmachev, A. V.; Baker, E. S.; Smith, R. D. Anal. Chem. 2016, 88 (18), 8949-8956.

(48) Prabhakaran, A.; Garimella, S. V. B.; Norheim, R.; Schimelfenig, C. E.; Prost, S. A.; Giberson, C.; Ibrahim, Y. M.; Smith, R. D. In Implementation of Array of Traps and Ion Elevators in Structures for Lossless Ion Manipulations (SLIM), Proceedings of the 66th ASMS Conference on Mass Spectrometry and Allied Topics, San Diego, California, June 3-7, 2018; San Diego, California, 2018.

(49) Pigman, W.; Isbell, H. S. Advances in Carbohydrate Chemistry 1968, 23, 11-57.

(50) Pfenninger, A.; Karas, M.; Finke, B.; Stahl, B. J. Am. Soc. Mass Spectrom. 2002, 13 (11), 1331-1340. 